\title{
Rossicasins A, B and Rosicaside F, Three New Phenylpropanoid Glycosides from Boschniakia rossica
}

\author{
Ming-Hwang SHYR, ${ }^{b}$ Tung-Hu TsaI, ${ }^{c}$ and Lie-Chwen LiN $*, a, d$ \\ ${ }^{a}$ National Research Institute of Chinese Medicine; Taipei, Taiwan: ${ }^{b}$ Department of Anesthesiology, Buddhist Tzu Chi \\ General Hospital; Hualien, 970, Taiwan: ' Institute of Traditional Medicine, National Yang-Ming University; Taipei, \\ Taiwan: and ${ }^{d}$ Graduate Institute of Integration Chinese and Western Medicine, China Medical University; Taichung, \\ Taiwan. Received August 26, 2005; accepted October 21, 2005
}

Three phenylpropanoid glycosides have been isolated, together with the known phenylpropanoid glycosides rossicaside A (4), B (5), E (6), and trans-p-coumaryl alcohol 1- $O$ - $\beta$-D-glucopyranosyl(1 $\rightarrow 4)$ - $\alpha$-L-rhamnopyra$\operatorname{nosyl}(1 \rightarrow 3)$ - $\beta$-D-glucopyranoside $(7)$, and an acylated oligosaccharide $\beta$-D-glucopyranosyl $(1 \rightarrow 4)$ - $\alpha$-L-rhamnopyranosyl-(1 $\rightarrow 3)-(4-O$-trans-caffeoyl)-D-glucopyranose) $(8)$, from the aqueous extract of Boschniakia rossica (CHAM. et Schlech.) Fedtsch. et Flerov. Spectroscopic evidence led to the assignments of their structures as trans-pcoumaryl-(6'-O- $\beta$-D-xylopyranosyl)- $O$ - $\beta$-D-glucopyranoside (1), trans- $p$-coumaryl-(6'- $O$ - $\alpha$-L-arabinopyranosyl)$O$ - $\beta$-D-glucopyranoside (2) and 2-(3,4-dihydroxyphenyl)- $R, S$-2-ethoxy-ethyl- $O$ - $\beta$-D-glucopyranosyl $(1 \rightarrow 4)$ - $\alpha$-Lrhamnopyranosyl $(1 \rightarrow 3)(4-O$-trans-caffeoyl)- $\beta$-D-glucopyranoside $(3)$, designated as rossicasin $A$, rossicasin $B$, and rossicaside $\mathrm{F}$, respectively. Compound 7 was identified from the degradation reaction and this is the first isolation from a natural source.

Key words Boschniakia rossica; Orobanchaceae; phenylpropanoid glycoside; rossicasin A; rossicasin B; rossicaside F

Boschniakia rossica (CHAm. et Schlech.) FEDTSCH. et FLEROV. (Orobanchaceae) is a parasitic plant growing on the root of plants of the genus Alnus (Betulaceae), as a substitute for Cistanchis Herba, a famous staminal tonic agent. ${ }^{1)}$ The crude extracts of $B$. rossica showed a variety of pharmacological activities including antitumor, ${ }^{2}$ anti-inflammatory, ${ }^{2)}$ antisenile, ${ }^{3)}$ antioxidative, and free radical scavenging activities. ${ }^{4)}$ Previous chemical studies of $B$. rossica have led to the isolation of a number of phenylpropanoid glycosides, iridoid glucosides, iridoid aglycones, and triterpenoids. ${ }^{5-8)}$ Further studies on this plant led to the isolation of three new phenylpropanoid glycosides. In this paper, we report the isolation and structures of these compounds.

\section{Results and Discussion}

The water-soluble fraction of the ethanolic extract of $B$. rossica was subjected to column chromatography by the procedure described in the Experimental section to yield seven phenylpropanoid glycosides (1-7) and an acylated oligosaccharide 8. Spectroscopic data obtained from compounds $\left.4,{ }^{5,6}\right)$ $5,{ }^{5,7)} \mathbf{6}^{5)}{ }^{5},{ }^{7)}$ and $\mathbf{8}^{6)}$ were in very good agreement with the literature data.

Rossicasin A (1) and rossicasin B (2) were found to be trans- $p$-coumaryl glycosides according to the ${ }^{1} \mathrm{H}$ - and ${ }^{13} \mathrm{C}$ NMR data. Compounds $\mathbf{1}$ and $\mathbf{2}$ had identical quasi-molecular ions at $m / z 443[\mathrm{M}-\mathrm{H}]^{-}$in ESI-MS and HR-FAB-MS $[\mathrm{M}+\mathrm{H}]^{+}$ions at $\mathrm{m} / \mathrm{z} 445.1715$ and 445.1709 , respectively, indicating the same molecular formula, $\mathrm{C}_{20} \mathrm{H}_{28} \mathrm{O}_{11}$. The ESIMS spectra of $\mathbf{1}$ and $\mathbf{2}$ showed only the same fragment ion at $\mathrm{m} / \mathrm{z} 311[\mathrm{M}-133]^{-}$, indicating a similar structure which loses a pentose $\left[\mathrm{C}_{5} \mathrm{O}_{4} \mathrm{H}_{9}\right]^{-}$mass unit from the molecular structure. In the ${ }^{1} \mathrm{H}-\mathrm{NMR}$ spectrum of 1 , signals at $\delta 4.29$ $(1 \mathrm{H}, \mathrm{dd}, J=12.5,6.5 \mathrm{~Hz}, \mathrm{Ha}-9), \delta 4.48(1 \mathrm{H}, \mathrm{dd}, J=12.5$, $6.0 \mathrm{~Hz}, \mathrm{Hb}-9), \delta 6.17(1 \mathrm{H}, \mathrm{dt}, J=16.0,6.5 \mathrm{~Hz}, \mathrm{H}-8), \delta 6.59$ $(1 \mathrm{H}, \mathrm{d}, J=16.0 \mathrm{~Hz}, \mathrm{H}-7$ ), and $\delta 6.74 / 7.27$ (each $2 \mathrm{H}, \mathrm{d}$, $J=8.5 \mathrm{~Hz}, \mathrm{H}-3,-5 / \mathrm{H}-2,-6)$ suggested the presence of transp-coumaryl moieties that were identical with those of 2 . In addition to the signals for the trans- $p$-coumaryl moiety, their
${ }^{1} \mathrm{H}-,{ }^{13} \mathrm{C}-\mathrm{NMR}$ spectra showed two sugar anomeric signals at $\delta_{\mathrm{H}} 4.37\left(\mathrm{~d}, J=8.0 \mathrm{~Hz}, \mathrm{H}-1^{\prime}\right) / \delta_{\mathrm{C}} 103.1(\mathrm{~d})$ and $\delta_{\mathrm{H}} 4.36(\mathrm{~d}$, $\left.J=7.0 \mathrm{~Hz}, \mathrm{H}-1^{\prime \prime}\right) / \delta_{\mathrm{C}} 105.5$ (d) for 1 , and $\delta_{\mathrm{H}} 4.37(\mathrm{~d}$, $\left.J=7.5 \mathrm{~Hz}, \mathrm{H}-1^{\prime}\right) / \delta_{\mathrm{C}} 103.2(\mathrm{~d})$ and $\delta_{\mathrm{H}} 4.35(\mathrm{~d}, J=7.0 \mathrm{~Hz}, \mathrm{H}-$ $\left.1^{\prime \prime}\right) / \delta_{\mathrm{C}} 105.2$ (d) for 2 . Combination of ${ }^{1} \mathrm{H}-{ }^{1} \mathrm{H}$ COSY, 1DTOCSY, and HMQC spectral data revealed that the sugar residues of 1 consisted of $\beta$-glucopyranose and $\beta$-xylopyranose and of 2 consisted of $\beta$-glucopyranose and $\alpha$-arabinopyranose. ${ }^{9)}$ Acid hydrolysis with $2 \mathrm{~N}_{2} \mathrm{SO}_{4}$ afforded Dglucose and D-xylose in 1 (identified by HPLC), and afforded
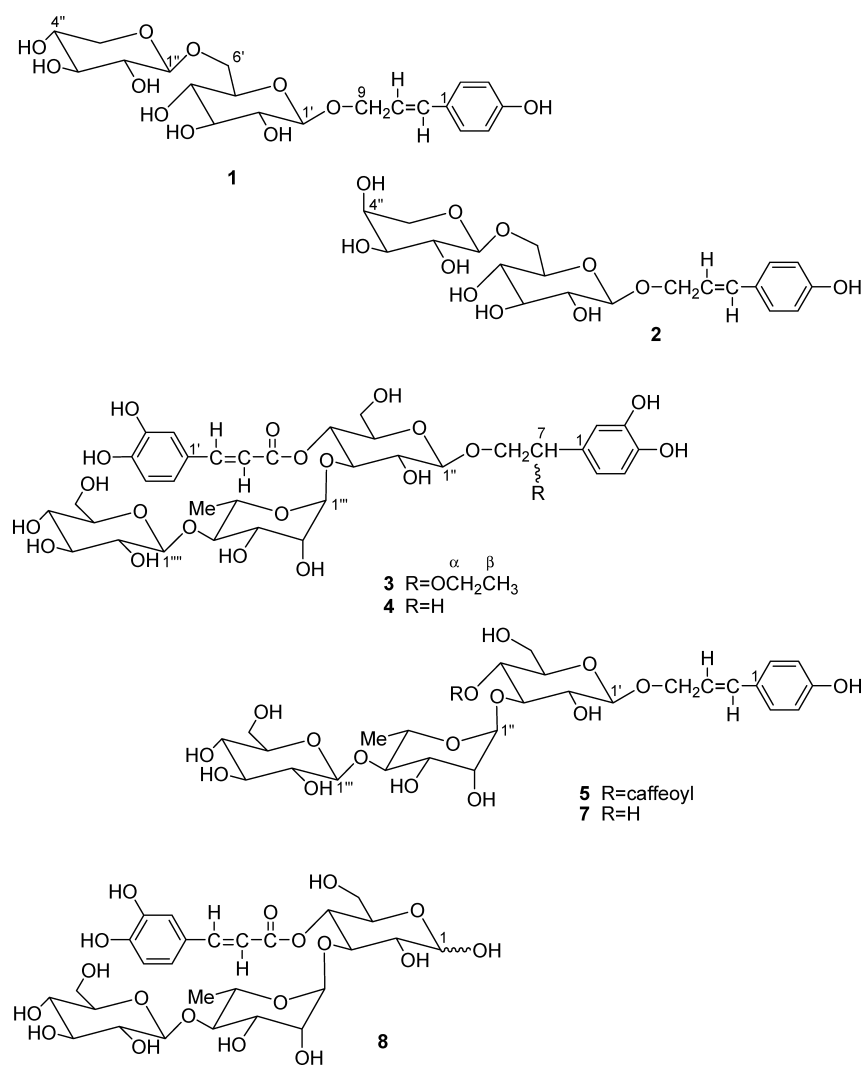

(C) 2006 Pharmaceutical Society of Japan 
D-glucose and L-arabinose in 2 (identified by HPLC). HMBC correlations of 1 from $\mathrm{H}-9 \mathrm{a} / \mathrm{b}$ to $\mathrm{C}-\mathrm{1}^{\prime}$ and from $\mathrm{H}-\mathrm{1}^{\prime}$ to $\mathrm{C}-9$ confirmed the attachment of a glucose unit to aglycone, and the position of the xylose unit was confirmed in a similar manner by correlations from $\mathrm{H}-\mathrm{1}^{\prime \prime}$ to $\mathrm{C}-\mathrm{6}^{\prime}$ and from $\mathrm{H}-\mathrm{6}^{\prime} \mathrm{a} / \mathrm{b}$ to $\mathrm{C}-1^{\prime \prime}$. On the basis of the above spectral data, we propose the structure of 1 as being trans- $p$-coumaryl- $\left(6^{\prime}-O-\beta\right.$-D-xylopyranosyl)- $O-\beta$-D-glucopyranoside. HMBC correlations of 2 from $\mathrm{H}-9 \mathrm{a} / \mathrm{b}$ to $\mathrm{C}-1^{\prime}$ and from $\mathrm{H}-1^{\prime}$ to $\mathrm{C}-9$ confirmed the attachment of a glucose unit to aglycone, and the position of the arabinose unit was confirmed in a similar manner by correlations from $\mathrm{H}-\mathrm{1}^{\prime \prime}$ to $\mathrm{C}-6^{\prime}$ and from $\mathrm{H}-6^{\prime} \mathrm{a} / \mathrm{b}$ to $\mathrm{C}-\mathrm{1}^{\prime \prime}$. On the basis of the above spectral data, we propose the structure of 2 as being trans- $p$-coumaryl-(6'-O- $\alpha$-L-arabinopyranosyl)-O$\beta$-D-glucopyranoside.

The new phenylpropanoid glycoside rossicaside F (3) showed a dirty green coloration with ferric chloride reagent, suggesting the presence of a phenolic hydroxyl group in the molecular structure. The ${ }^{1} \mathrm{H}-\mathrm{NMR}$ spectrum of $\mathbf{3}$ showed the presence of three anomeric protons at $\delta 4.44 / 4.45$ (total $1 \mathrm{H}$, each d, $\left.J=8.0 \mathrm{~Hz}, \mathrm{H}-1^{\prime \prime}\right), 4.48\left(1 \mathrm{H}, \mathrm{d}, J=7.0 \mathrm{~Hz}, \mathrm{H}-1^{\prime \prime \prime \prime}\right)$, and $5.26(1 \mathrm{H}$, br s, H-1"' $)$, consistent with the presence of two $\beta$ glucose and an $\alpha$-rhamnose unit, and ethoxy signals at $\delta$ $3.43(2 \mathrm{H}, \mathrm{m})$ and $\delta 1.18(3 \mathrm{H}, \mathrm{t}, J=7.5 \mathrm{~Hz})$. A set of signals of an aromatic ABX system $(\delta 6.97,6.80,7.07)$ and trans $\alpha$, $\beta$ unsaturated protons $(\delta 6.26,7.59$, each d, $J=15.5 \mathrm{~Hz}, \mathrm{H}-$ $\left.8^{\prime}, 7^{\prime}\right)$ suggested the presence of trans-caffeic acid. Other ABX aromatic protons $(\delta 6.67,6.76,6.79)$, oxygenated methylene protons $\left(\delta 3.68,3.85, \mathrm{H}_{2}-8\right)$, and an oxygenated methine proton $\left(\delta 4.48\right.$, overlap with $\left.\mathrm{H}-1^{\prime \prime \prime \prime}\right)$ were assigned to the $\beta, 3,4$-trihydroxy-phenethyl alcohol moiety. The ${ }^{1} \mathrm{H}$ - and ${ }^{13} \mathrm{C}$-NMR spectra of $\mathbf{3}$ were similar to those of rossicaside A (4) ${ }^{5)}$ except for the presence of ethoxy signals, suggesting that $\mathbf{3}$ is an ethyl ether derivative of rossicaside A. The mass spectrum of 3 showed a quasi-molecular ion at $\mathrm{m} / \mathrm{z} 829$ $[\mathrm{M}-\mathrm{H}]^{-}$, corresponding to an additional ethoxyl group as compared to rossicaside A. HMBC correlations from C-7 to $\mathrm{H}-2, \mathrm{H}-6$, and $\mathrm{H}_{2}-\alpha$ confirmed the attachment of an ethyl ether unit to $\mathrm{C}-7$. Though 3 only gave a spot on TLC, the ${ }^{13} \mathrm{C}-\mathrm{NMR}$ spectral data of $\mathbf{3}$ showed two kinds of chemical shift for each carbon in the vicinity of the asymmetric $\mathrm{C}-7$, such as $\mathrm{C}-1, \mathrm{C}-6, \mathrm{C}-7, \mathrm{C}-8, \mathrm{C}-1^{\prime}$, C- $\alpha$ and $\mathrm{C}-\beta$. These findings indicated that 3 existed as epimers at the $\beta$-C of the phenethyl alcohol moiety $\left(R, S\right.$ - $\beta$-OEt) like campneoside $\mathrm{I}^{10}{ }^{10}$ Accordingly, compound 3 was characterized as 2-(3,4-dihydroxyphenyl)- $R, S$-2-ethoxy-ethyl- $O$ - $\beta$-D-glucopyranosyl$(1 \rightarrow 4)$ - $\alpha$-L-rhamnopyranosyl $(1 \rightarrow 3)(4-O$-trans-caffeoyl $)-\beta$ D-glucopyranoside.

Compound 7 had the molecular formula $\mathrm{C}_{27} \mathrm{H}_{40} \mathrm{O}_{16}$ as determined by a combination of ESI-MS $\left(m / z 619[\mathrm{M}-\mathrm{H}]^{-}\right)$, ${ }^{13} \mathrm{C}-\mathrm{NMR}$, and DEPT spectra. The ${ }^{1} \mathrm{H}$ - and ${ }^{13} \mathrm{C}-\mathrm{NMR}$ spectral data of 7 were very similar with those of rossicaside $\mathrm{B}$ $(5)^{5)}$ except for the disappearance of the caffeoyl group, as shown in the Experimental section. This means that 7 has a $p$-coumaryl, a rhamnose, and two glucose moieties. From the similarity of these spectral data, the linkage positions of the coumaryl, rhamnose, and two glucose moieties were concluded to be rossicaside B (5). HMBC correlations from $\mathrm{C}-1^{\prime}$ $(\delta 102.9)$ to $\mathrm{H}-9 \mathrm{a} / 9 \mathrm{~b}(\delta 4.29 / 4.49)$, from $\mathrm{C}-1^{\prime \prime}(\delta 102.4)$ to H-3' $(\delta 3.53)$, and from C-1"' $(\delta$ 105.5) to H-4" $(\delta$ 3.64) also supported the above deductions. Therefore, the structure of 7 was determined as trans- $p$-coumaryl alcohol 1- $O$ - $\beta$-D-glucopyranosyl $(1 \rightarrow 4)$ - $\alpha$-L-rhamnopyranosyl $(1 \rightarrow 3)$ - $\beta$-D-glucopyranoside. Compound 7 has been identified from the degradation reaction, ${ }^{7}$ ) and this is the first isolation from a natural source.

\section{Experimental}

Apparatus All melting points were determined on a Yanagimoto micromelting point apparatus and are uncorrected. IR spectra were obtained as $\mathrm{KBr}$ pellets or film on a Nicolet Avatar 320 IR spectrometer. UV spectra were measured on a Hitachi U-3200 spectrophotometer in $\mathrm{MeOH} .{ }^{1} \mathrm{H}-,{ }^{13} \mathrm{C}-$ and 2D-NMR spectra were measured with a Varian Inova-500 spectrometer with deuterated solvents as internal standard. ESI-MS and HR-FAB-MS were recorded on Finnigan LCQ and Finnigan/Thermo Quest MAT spectrometers, respectively. HPLC analysis was performed using a Shimadzu LC-8A or LC-10AT vp pump and SPD-10A vp UV-Vis detector or RIA$10 \mathrm{~A}$ refractive index detector.

Plant Material The whole plant of Boschniakia rossica was purchased in Taipei, Taiwan, and verified by Mr. Hsi-Yu Chang, director of Feng Li Co., Inc., Taipei, Taiwan. A voucher specimen is deposited in the National Research Institute of Chinese Medicine, ROC.

Isolation The whole herb of B. rossica $(9.6 \mathrm{~kg})$ was extracted with $95 \%$ EtOH $(601 \times 4)$ under reflux. The ethanolic extracts were combined and concentrated under vaccum to a volume of 1.51 . The ethanolic extract was then partitioned successively between $\mathrm{H}_{2} \mathrm{O}$ and EtOAc, followed by $n$-BuOH (each $11 \times 3)$. A portion $(200 \mathrm{~g})$ of the $\mathrm{H}_{2} \mathrm{O}$ extract $(700 \mathrm{~g})$ was subjected to column chromatography over Diaion $\mathrm{HP}-20(10 \mathrm{~cm} \times 50 \mathrm{~cm})$ with $\mathrm{H}_{2} \mathrm{O}, 20 \%$ $\mathrm{MeOH} / \mathrm{H}_{2} \mathrm{O}, 50 \% \mathrm{MeOH} / \mathrm{H}_{2} \mathrm{O}$, and $\mathrm{MeOH}$ as the eluting solvents to give 4 fractions. Fr. $2(40 \mathrm{~g})$ was rechromatographed over Sephadex LH-20 with aqueous $\mathrm{MeOH}(0-20 \%)$ and further purified by Cosmosil $\mathrm{C}_{18}$ OPN 140 (20- $40 \% \mathrm{MeOH}$ in $\mathrm{H}_{2} \mathrm{O}$ ) to give Fr. 2-1 and 2-2. Fr. 2-1 was recrystallized with $\mathrm{H}_{2} \mathrm{O}$ to give $8(876 \mathrm{mg})$. Fr. 2-2 was further purified with semipreparative HPLC (column: Cosmosil $\mathrm{NH}_{2}, 5 \mu \mathrm{m}, 25 \times 250 \mathrm{~mm}$; mobile phase: $80 \%$ $\mathrm{CH}_{3} \mathrm{CN} / \mathrm{H}_{2} \mathrm{O}$; flow rate: $16 \mathrm{ml} / \mathrm{min}$, detector: $\mathrm{UV} 254 \mathrm{~nm}$ ) to give compounds $1(65 \mathrm{mg}), 2(13 \mathrm{mg})$ and $7(190 \mathrm{mg})$. Fr. $3(16 \mathrm{~g})$ was chromatographyed over a Sephadex LH-20 $\left(0-60 \% \mathrm{MeOH}\right.$ in $\left.\mathrm{H}_{2} \mathrm{O}\right)$ to give Fr. 3-1-39. Fr. 3-5 was further purified with semipreparative HPLC (column: Inertsil 10 ODS, $22 \times 250 \mathrm{~mm}$; mobile phase: $18 \% \mathrm{CH}_{3} \mathrm{CN} / \mathrm{H}_{2} \mathrm{O}$; flow rate: $16 \mathrm{ml} / \mathrm{min}$, detector: UV $254 \mathrm{~nm})$ to give compounds $3(35 \mathrm{mg})$ and 4 $(125 \mathrm{mg})$. Repeated chromatography of fraction Fr. 3-7 over Sephadex LH$20(\mathrm{MeOH})$ and semipreparative HPLC (column: Inertsil 10 ODS, $22 \times 250 \mathrm{~mm}$; mobile phase: $50 \% \mathrm{MeOH} / \mathrm{H}_{2} \mathrm{O}$; flow rate: $15 \mathrm{ml} / \mathrm{min}$, detector: UV $254 \mathrm{~nm}$ ) yielded $5(728 \mathrm{mg})$ and $\mathbf{6}(590 \mathrm{mg})$.

Rossicasin A (1) Colorless needles $(\mathrm{MeOH}), \mathrm{mp} 168-170^{\circ} \mathrm{C} .[\alpha]_{\mathrm{D}}^{23}$ $-92.5^{\circ}\left(c=0.4, \mathrm{H}_{2} \mathrm{O}\right)$. UV $\lambda_{\max }(\mathrm{MeOH}) \mathrm{nm}(\varepsilon): 263$ (4.36). IR $v_{\max }^{\mathrm{KBr}} \mathrm{cm}^{-1}$ : $3455,3322(\mathrm{OH}), 1605,1520(\mathrm{C}=\mathrm{C}), 1451,1398,1351,1035,1005 .{ }^{1} \mathrm{H}-$ NMR $\left(\mathrm{CD}_{3} \mathrm{OD}\right) \delta: 3.22\left(1 \mathrm{H}, \mathrm{t}, J=11.0 \mathrm{~Hz}, \mathrm{Ha}-5^{\prime \prime}\right), 3.25\left(2 \mathrm{H}, \mathrm{m}, \mathrm{H}-2^{\prime},-2^{\prime \prime}\right)$, $3.35\left(1 \mathrm{H}, \mathrm{t}, J=9.0 \mathrm{~Hz}, \mathrm{H}-3^{\prime \prime}\right), 3.37\left(2 \mathrm{H}, \mathrm{m}, \mathrm{H}-3^{\prime}, 4^{\prime}\right), 3.45\left(1 \mathrm{H}, \mathrm{m}, \mathrm{H}-5^{\prime}\right)$, $3.52\left(1 \mathrm{H}, \mathrm{td}, J=9.5,5.5 \mathrm{~Hz}, \mathrm{H}-4^{\prime \prime}\right), 3.76\left(1 \mathrm{H}, \mathrm{dd}, J=11.0,5.5 \mathrm{~Hz}, \mathrm{Ha}-6^{\prime}\right)$ $3.89\left(1 \mathrm{H}, \mathrm{dd}, J=11.5,5.5 \mathrm{~Hz}, \mathrm{Hb}-5^{\prime \prime}\right), 4.11\left(1 \mathrm{H}, \mathrm{dd}, J=11.0,1.5 \mathrm{~Hz}, \mathrm{Hb}-6^{\prime}\right)$, $4.29(1 \mathrm{H}, \mathrm{dd}, J=12.5,6.5 \mathrm{~Hz}, \mathrm{Ha}-9), 4.36\left(1 \mathrm{H}, \mathrm{d}, J=7.0 \mathrm{~Hz}, \mathrm{H}-1^{\prime \prime}\right), 4.37$ $\left(1 \mathrm{H}, \mathrm{d}, J=8.0 \mathrm{~Hz}, \mathrm{H}-1^{\prime}\right), 4.48(1 \mathrm{H}, \mathrm{dd}, J=12.5,6.0 \mathrm{~Hz}, \mathrm{Hb}-9), 6.17(1 \mathrm{H}, \mathrm{dt}$, $J=16.0,6.5 \mathrm{~Hz}, \mathrm{H}-8), 6.59(1 \mathrm{H}, \mathrm{d}, J=16.0 \mathrm{~Hz}, \mathrm{H}-7), 6.74(2 \mathrm{H}, \mathrm{d}, J=8.5 \mathrm{~Hz}$, $\mathrm{H}-3,5), 7.27(2 \mathrm{H}, \mathrm{d}, J=8.5 \mathrm{~Hz}, \mathrm{H}-2,6) .{ }^{13} \mathrm{C}-\mathrm{NMR}\left(\mathrm{CD}_{3} \mathrm{OD}\right) \delta: 66.9\left(\mathrm{C}-5^{\prime \prime}\right)$, 69.7 (C-6'), $71.1\left(\mathrm{C}-4^{\prime \prime}\right), 71.2$ (C-9), $71.4\left(\mathrm{C}-4^{\prime}\right), 74.8\left(\mathrm{C}-2^{\prime}\right), 75.0\left(\mathrm{C}-2^{\prime \prime}\right)$, 76.9 (C-5'), 77.7 (C-3"), 77.9 (C-3'), $103.1\left(\mathrm{C}-1^{\prime}\right), 105.5$ (C-1"), 116.3 (C-3, 5), 123.3 (C-8), 128.9 (C-2, 6), 129.7 (C-1), 134.3 (C-7), 158.4 (C-4). ESIMS $m / z: 443[\mathrm{M}-\mathrm{H}]^{-}, 311[\mathrm{M}-133]^{-}$. HR-FAB-MS $\mathrm{m} / \mathrm{z} \quad 445.1715$ $[\mathrm{M}+1]^{+}\left(\right.$Calcd 445.1710 for $\left.\mathrm{C}_{20} \mathrm{H}_{29} \mathrm{O}_{11}\right)$.

Acid Hydrolysis of 1 A solution of $1(5 \mathrm{mg})$ in $2 \mathrm{~N} \mathrm{H}_{2} \mathrm{SO}_{4}(3 \mathrm{ml})$ was refluxed in a water bath for $2 \mathrm{~h} . \mathrm{H}_{2} \mathrm{O}$ was added to the solution, the mixture washed with $\mathrm{CHCl}_{3}$, the aqueous phase neutralized with $\mathrm{BaCO}_{3}$, and then the precipitate was filtered off. The filtrate was concentrated and examined by HPLC (Phenomenex Luna $5 \mu \mathrm{NH}_{2}, 250 \times 4.6 \mathrm{~mm}, 65 \%$ acetonitrile $/ \mathrm{H}_{2} \mathrm{O}$, $1.2 \mathrm{ml} / \mathrm{min}$, RI detector). D-glucose $\left(t_{\mathrm{R}}=4.50 \mathrm{~min}\right)$ and D-xylose $\left(t_{\mathrm{R}} 4.03 \mathrm{~min}\right)$ were detected by comparing them with the retention times $\left(t_{\mathrm{R}}\right)$ of authentic samples.

Rossicasin B (2) Brown syrup. $[\alpha]_{\mathrm{D}}^{23}-51.7^{\circ}\left(c=0.29, \mathrm{H}_{2} \mathrm{O}\right)$. UV $\lambda_{\max }$ $(\mathrm{MeOH}) \mathrm{nm}(\varepsilon): 263(4.02)$. IR $v_{\max }^{\mathrm{KBr}} \mathrm{cm}^{-1}: 3395(\mathrm{OH}), 1609,1514,1435$, 1372, 1062, $1009(\mathrm{C}=\mathrm{C}) .{ }^{1} \mathrm{H}-\mathrm{NMR}\left(\mathrm{CD}_{3} \mathrm{OD}\right) \delta: 3.24(1 \mathrm{H}, \mathrm{t}, J=8.0 \mathrm{~Hz}, \mathrm{H}-$ $\left.2^{\prime}\right), 3.36\left(2 \mathrm{H}, \mathrm{m}, \mathrm{H}-3^{\prime},-4^{\prime}\right), 3.45\left(1 \mathrm{H}, \mathrm{m}, \mathrm{H}-5^{\prime}\right), 3.54\left(2 \mathrm{H}, \mathrm{m}, \mathrm{H}-3^{\prime \prime}, \mathrm{Ha}-5^{\prime \prime}\right)$, $3.62\left(1 \mathrm{H}, \mathrm{dd}, J=9.0,6.5 \mathrm{~Hz}, \mathrm{H}-2^{\prime \prime}\right), 3.75\left(1 \mathrm{H}, \mathrm{dd}, J=11.5,5.5 \mathrm{~Hz}, \mathrm{Ha}-6^{\prime}\right)$, $3.81\left(1 \mathrm{H}, \mathrm{br} \mathrm{s}, \mathrm{H}-4^{\prime \prime}\right), 3.88\left(1 \mathrm{H}, \mathrm{dd}, J=12.5,3.5 \mathrm{~Hz}, \mathrm{Hb}-5^{\prime \prime}\right), 4.12(1 \mathrm{H}$, dd, 
$\left.J=11.5,2.0 \mathrm{~Hz}, \mathrm{Hb}-6^{\prime}\right), 4.29(1 \mathrm{H}, \mathrm{dd}, J=12.5,7.0 \mathrm{~Hz}, \mathrm{Ha}-9), 4.35(1 \mathrm{H}, \mathrm{d}$ $\left.J=7.0 \mathrm{~Hz}, \mathrm{H}-1^{\prime \prime}\right), 4.37\left(1 \mathrm{H}, \mathrm{d}, J=7.5 \mathrm{~Hz}, \mathrm{H}-1^{\prime}\right), 4.48(1 \mathrm{H}, \mathrm{dd}, J=12.5$, $6.5 \mathrm{~Hz}, \mathrm{Hb}-9), 6.17(1 \mathrm{H}, \mathrm{dt}, J=16.0,6.5 \mathrm{~Hz}, \mathrm{H}-8), 6.60(1 \mathrm{H}, \mathrm{d}, J=16.0 \mathrm{~Hz}$, $\mathrm{H}-7), 6.74(2 \mathrm{H}, \mathrm{d}, J=8.5 \mathrm{~Hz}, \mathrm{H}-3,5), 7.27(2 \mathrm{H}, \mathrm{d}, J=8.5 \mathrm{~Hz}, \mathrm{H}-2,6) .{ }^{13} \mathrm{C}-$ NMR (CD $\mathrm{OD}) \delta: 66.7\left(\mathrm{C}-5^{\prime \prime}\right), 69.5\left(\mathrm{C}-6^{\prime}, 4^{\prime \prime}\right), 71.2(\mathrm{C}-9), 71.7$ (C-4'), 72.4 (C-2"), $74.2\left(\mathrm{C}-3^{\prime \prime}\right), 75.1\left(\mathrm{C}-2^{\prime}\right), 76.9\left(\mathrm{C}-5^{\prime}\right), 78.0\left(\mathrm{C}-3^{\prime}\right), 103.2\left(\mathrm{C}-1^{\prime}\right), 105.2$ (C-1"), 116.3 (C-3, 5), 123.4 (C-8), 128.9 (C-2, 6), 129.8 (C-1), 134.1 (C-7), 158.4 (C-4). ESI-MS $m / z: 443[\mathrm{M}-\mathrm{H}]^{-}, 311$ [M-133] $^{-}$. HR-FAB-MS $m / z$ $445.1709[\mathrm{M}+1]^{+}$(Calcd 445.1710 for $\left.\mathrm{C}_{20} \mathrm{H}_{29} \mathrm{O}_{11}\right)$.

Acid Hydrolysis of 2 A mixture of $2(3 \mathrm{mg})$ and $2 \mathrm{~N}_{2} \mathrm{SO}_{4}(3 \mathrm{ml})$ was heated in a water bath for $2 \mathrm{~h}$. The products D-glucose $\left(t_{\mathrm{R}}=4.48 \mathrm{~min}\right)$ and $\mathrm{L}-$ arabinose $\left(t_{\mathrm{R}}=3.68 \mathrm{~min}\right)$ were isolated in the HPLC analysis, as described for 1 .

Rossicaside F (3) Brown syrup. $[\alpha]_{\mathrm{D}}^{23}-70.9^{\circ}\left(c=0.79, \mathrm{H}_{2} \mathrm{O}\right)$. UV $\lambda_{\max }$ $(\mathrm{MeOH}) \mathrm{nm}(\varepsilon): 333$ (4.18), 290 sh. (4.01), 219 (4.21). IR $v_{\max }^{\mathrm{KBr}} \mathrm{cm}^{-1}: 3390$ $(\mathrm{OH}), 1698(\mathrm{C}=\mathrm{O}), 1630,1604,1520(\mathrm{C}=\mathrm{C}), 1170,1062,1020 .{ }^{1} \mathrm{H}-\mathrm{NMR}$ $\left(\mathrm{CD}_{3} \mathrm{OD}\right) \delta: 1.18(3 \mathrm{H}, \mathrm{t}, J=7.5 \mathrm{~Hz}, \mathrm{H}-\beta), 1.20\left(3 \mathrm{H}, \mathrm{d}, J=6.5 \mathrm{~Hz}, \mathrm{H}-6^{\prime \prime \prime}\right)$, $3.05\left(1 \mathrm{H}, \mathrm{t}, J=7.5 \mathrm{~Hz}, \mathrm{H}-2^{\prime \prime \prime \prime}\right), 3.43\left(3 \mathrm{H}, \mathrm{m}, \mathrm{H}-\alpha, 2^{\prime \prime}\right), 3.68(2 \mathrm{H}, \mathrm{m}, \mathrm{Ha}-8, \mathrm{H}-$ $\left.5^{\prime \prime \prime}\right), 3.81\left(1 \mathrm{H}, \mathrm{t}, J=9.5 \mathrm{~Hz}, \mathrm{H}-3^{\prime \prime}\right), 3.85(1 \mathrm{H}, \mathrm{m}, \mathrm{Hb}-8), 4.44 / 4.45$ (total $1 \mathrm{H}$, each d, $\left.J=8.0 \mathrm{~Hz}, \mathrm{H}-1^{\prime \prime}\right), 4.48\left(2 \mathrm{H}, \mathrm{m}, \mathrm{H}-1^{\prime \prime \prime \prime}, 7\right), 4.94(1 \mathrm{H}, \mathrm{t}, J=9.0 \mathrm{~Hz}, \mathrm{H}-$ 4"), $5.26\left(1 \mathrm{H}, \mathrm{s}, \mathrm{H}-1^{\prime \prime \prime}\right), 6.26\left(1 \mathrm{H}, \mathrm{d}, J=15.5 \mathrm{~Hz}, \mathrm{H}-8^{\prime}\right), 6.67(1 \mathrm{H}, \mathrm{d}$ $J=8.0 \mathrm{~Hz}, \mathrm{H}-6), 6.76(1 \mathrm{H}, \mathrm{d}, J=8.0 \mathrm{~Hz}, \mathrm{H}-5), 6.79(1 \mathrm{H}, \mathrm{s}, \mathrm{H}-2), 6.80(1 \mathrm{H}, \mathrm{d}$, $\left.J=8.0 \mathrm{~Hz}, \mathrm{H}-5^{\prime}\right), 6.97\left(1 \mathrm{H}, \mathrm{d}, J=8.0 \mathrm{~Hz}, \mathrm{H}-6^{\prime}\right), 7.07\left(1 \mathrm{H}, \mathrm{s}, \mathrm{H}-2^{\prime}\right), 7.59(1 \mathrm{H}$, d, $\left.J=15.5 \mathrm{~Hz}, \mathrm{H}-7^{\prime}\right) .{ }^{13} \mathrm{C}-\mathrm{NMR}\left(\mathrm{CD}_{3} \mathrm{OD}\right) \delta: 15.4 / 15.5(\mathrm{C}-\beta), 18.5\left(\mathrm{C}-6^{\prime \prime \prime}\right)$, 62.3 (C-6"), 62.8 (C-6"' $), 65.0 / 65.1(\mathrm{C}-\alpha), 68.8\left(\mathrm{C}-5^{\prime \prime \prime}\right), 70.4\left(\mathrm{C}-4^{\prime \prime}\right), 71.5(\mathrm{C}-$ $\left.4^{\prime \prime \prime \prime}\right), 72.1$ (C-3"'), 72.2 (C-2"'), 75.1/76.6 (C-8), 75.8 (C-2'"'), 76.0 (C-5"), 76.4 (C-2"), 77.8 (C-5'"'), 78.1 (C-3"'"), 80.9 (C-3"), 82.5/82.6 (C-7), 83.5 (C-4"'), 102.4 (C-1"'), 103.9/104.1 (C-1"), 105.5 (C-1"'"), 114.6 (C-8'), 114.6/114.9 (C-2), 115.4 (C-2'), 116.2/116.3 (C-5), $116.6 \quad\left(\mathrm{C}-5^{\prime}\right)$, 119.7/119.8 (C-6), 123.4 (C-6'), $127.7\left(\mathrm{C}-1^{\prime}\right), 131.4 / 131.8$ (C-1), 146.3 (C4), 146.5 (C-3), 147.7 (C-3'), 148.2 (C-7'), 149.8 (C-4'), 168.3 (C-9'). ESIMS $m / z: 829[\mathrm{M}-\mathrm{H}]^{-}$. HR-FAB-MS $m / z: 831.2924[\mathrm{M}+1]^{+}$(Calcd 831.2923 for $\mathrm{C}_{37} \mathrm{H}_{51} \mathrm{O}_{21}$ ).

trans-p-Coumaryl Alcohol 1- $O$ - $\beta$-Glucopyranosyl $(1 \rightarrow 4)$ - $\alpha$-rhamnopyranosyl $(\mathbf{1} \rightarrow \mathbf{3})$ - $\boldsymbol{\beta}$-glucopyranoside (7) Brown syrup. UV $\lambda_{\max }(\mathrm{MeOH})$ $\mathrm{nm}(\varepsilon): 263$ (4.12). IR $v_{\max }^{\mathrm{KBr}} \mathrm{cm}^{-1}: 3390(\mathrm{OH}), 1605,1514(\mathrm{C}=\mathrm{C}), 1235$, 1078, 1030. ${ }^{1} \mathrm{H}-\mathrm{NMR}\left(\mathrm{CD}_{3} \mathrm{OD}\right) \delta: 1.33\left(3 \mathrm{H}, \mathrm{d}, J=6.0 \mathrm{~Hz}, \mathrm{H}-6{ }^{\prime \prime}\right), 3.23(1 \mathrm{H}$, t, $\left.J=9.0 \mathrm{~Hz}, \mathrm{H}-2^{\prime \prime \prime}\right), 3.53\left(1 \mathrm{H}, \mathrm{t}, J=9.0 \mathrm{~Hz}, \mathrm{H}-3^{\prime}\right), 3.64(1 \mathrm{H}, \mathrm{t}, J=9.0 \mathrm{~Hz}, \mathrm{H}-$
4"), $3.72\left(2 \mathrm{H}, \mathrm{m}, \mathrm{Ha}-6^{\prime}, 6^{\prime \prime \prime}\right), 3.85\left(1 \mathrm{H}, \mathrm{dd}, J=11.5,2.0 \mathrm{~Hz}, \mathrm{Hb}-6^{\prime \prime \prime}\right), 3.89$ $\left(1 \mathrm{H}, \mathrm{dd}, J=11.5,2.0 \mathrm{~Hz}, \mathrm{Hb}-6^{\prime}\right), 3.96\left(1 \mathrm{H}, \mathrm{dd}, J=9.5,3.0 \mathrm{~Hz}, \mathrm{H}-3^{\prime \prime}\right), 3.99$ $\left(1 \mathrm{H}\right.$, br s, H-2"), $4.09\left(1 \mathrm{H}, \mathrm{m}, \mathrm{H}-5^{\prime \prime}\right), 4.29(1 \mathrm{H}, \mathrm{dd}, J=12.5,6.5 \mathrm{~Hz}, \mathrm{Ha}-9)$, $4.38\left(1 \mathrm{H}, \mathrm{d}, J=7.0 \mathrm{~Hz}, \mathrm{H}-1^{\prime}\right), 4.49(1 \mathrm{H}, \mathrm{dd}, J=12.5,6.5 \mathrm{~Hz}, \mathrm{Hb}-9), 4.60$ $\left(1 \mathrm{H}, \mathrm{d}, J=8.0 \mathrm{~Hz}, \mathrm{H}-1^{\prime \prime \prime}\right), 5.18\left(1 \mathrm{H}, \mathrm{s}, \mathrm{H}-1^{\prime \prime}\right), 6.17(1 \mathrm{H}, \mathrm{dt}, J=16.0,6.5 \mathrm{~Hz}$, $\mathrm{H}-8), 6.60(1 \mathrm{H}, \mathrm{d}, J=16.0 \mathrm{~Hz}, \mathrm{H}-7), 6.75(2 \mathrm{H}, \mathrm{d}, J=8.5 \mathrm{~Hz}, \mathrm{H}-3,5), 7.26$ $(2 \mathrm{H}, \mathrm{d}, J=8.5 \mathrm{~Hz}, \mathrm{H}-2,6) .{ }^{13} \mathrm{C}-\mathrm{NMR}\left(\mathrm{CD}_{3} \mathrm{OD}\right) \delta: 18.0\left(\mathrm{C}-6^{\prime \prime}\right), 62.6\left(\mathrm{C}-6{ }^{\prime}\right.$, $\left.6^{\prime \prime \prime}\right), 68.6\left(\mathrm{C}-5^{\prime \prime}\right), 70.1\left(\mathrm{C}-4^{\prime}\right), 71.2(\mathrm{C}-9), 71.4\left(\mathrm{C}-4^{\prime \prime \prime}\right), 72.1\left(\mathrm{C}-2^{\prime \prime}\right), 72.2(\mathrm{C}-$ $\left.3^{\prime \prime}\right), 75.6\left(\mathrm{C}-2^{\prime}\right), 76.0\left(\mathrm{C}-2^{\prime \prime \prime}\right), 77.7$ (C-5'), $77.9\left(\mathrm{C}-5^{\prime \prime \prime}\right), 78.1$ (C-3"'), 83.5 (C$\left.4^{\prime \prime}\right), 84.1\left(\mathrm{C}-3^{\prime}\right), 102.4\left(\mathrm{C}-1^{\prime \prime}\right), 102.9\left(\mathrm{C}-1^{\prime}\right), 105.2\left(\mathrm{C}-1^{\prime \prime \prime}\right), 116.3(\mathrm{C}-3,5)$, 123.3 (C-8), 128.8 (C-2, 6), 129.7 (C-1), 134.1 (C-7), 158.3 (C-4). ESI-MS $m / z: 619[\mathrm{M}-\mathrm{H}]^{-}$.

Acknowledgement We are grateful to the National Science Council, the Republic of China, for support of this research under Grant NSC 93-2113M-077-001.

\section{References}

1) Juangsu New Medical College. Zhong Yao Da Ci Dian "Dictionary of Chinese Materia Medica," Shanghai Scientific and Technological Publishers, Shanghai, 1979, p. 1583.

2) Yin Z. Z., Jin H. L., Yin X. Z., Li, T. Z., Quan J. S., Jin Z. N., World J. Gastroentero., 12, 812-818 (2000).

3) Tsuda T., Liu Y. Z., Sugaya A., Katoh K., Hori K., Tanaka S., Nomura M., Sugaya E., J. Ethnopharmacology, 44, 67-71 (1994).

4) Tsuda T., Sugaya A., Liu Y. Z., Katoh K., Tanaka H., Kawazura H., Sugaya E., Kusai M., Kohno M., J. Ethnopharmacology, 41, 85-90 (1994).

5) Lin L. C., Chen K. T., Chin. Pharm. J., 56, 77-85 (2004).

6) Konishi T., Narumi Y., Watanabe K., Kiyosawa S., Shoji J., Chem. Pharm. Bull., 35, 4155-4161 (1987).

7) Konishi T., Shoji J., Chem. Pharm. Bull., 29, 2807-2815 (1981).

8) Yim S. H., Kim H. J., Liu Y. Z., Lee I. S., Chem. Pharm. Bull., 52, 289-290 (2004).

9) Tolonen A., Pakonen M., Hohtola A., Jalonen J., Chem. Pharm. Bull., 51, 467-470 (2003).

10) Imakura Y., Kobayashi S., Mima A., Phytochemistry, 24, 139-146 (1985). 